\title{
Influence of Implantation Period on Bioactivity on the Surface of Ti-6Al-4V Implant Model Made by Selective Laser Melting
}

\author{
Asuka Kutsukake, Azusa Seki and Yoshinori Yoshida
}

\author{
Asuka Kutsukake. Center for Advanced and Smart Die Engineering Technology (G-CADET), Gifu University, Tokai National Higher \\ Education and Research System, Japan \\ Corresponding author: y3921009@edu.gifu-u.ac.jp \\ Azusa Seki. HAMRI CO., LTD., Japan \\ Yoshinori Yoshida. Center for Advanced and Smart Die Engineering Technology (G-CADET), Gifu University, Tokai National \\ Higher Education and Research System, Japan
}

\begin{abstract}
Square pole implant models of Ti-6Al-4V were fabricated by selective laser melting (SLM) and osteoconductivity was investigated on their surface. The models have 3 types of surfaces; top surface, side surface, and polished surface. The surfaces have each different surface roughness and the influence of the roughness on the osteoconductivity was observed in-vivo experiment. The models were implanted in rat femurs and observed after 2 and 8 weeks. We observed the amount of hard tissue produced on the surfaces in the cut-off cross section of the femurs with the model by means of an optical microscope and bone-implant contact ratio $\left(R_{B-I}\right)$ was evaluated. As the result, in the case of 2 weeks-raised rats, the $R_{B-I}$ of the polished surface was the highest of all surfaces. The $R_{B-I}$ of the surface was however the lowest and that of the top surface was the highest in the case of 8 weeks-raised rats.
\end{abstract}

Keywords. Additive Manufacturing, Selective Laser Melting, Biomedical Implant, Bioactivity

\section{Introduction}

Additive manufacturing (AM) technology enables to make products with complex shapes. The products are automatically fabricated based on the sliced data created from 3D CAD. By using the technology, it is possible to make products with special shape that could not be made with conventional methods such as machining and forging. The AM technology is an effective production method for medical devices, especially for custom-made implants. The shape and dimension of the product should be designed depending on patient's skeleton. It is possible to manufacture the implants designed for each patient based on the 3D CAD constructed from CT images. Selective laser melting (SLM) is one of powder bed fusion process in the AM technology. As-fabricated surface is usually rough, and the surface is cut or polished in the finishing process. When the shape of the implant fabricated by AM is small or complicated, it is difficult to polish the surface. In the case of the lattice structure, for example, there are many small pillars inside, as-fabricated surface of the pillars is used without polishing.

The implants used into bone require not only in-vivo safety but also bioactivity. The bioactivity of implant surface depends on chemical coatings, surface asperity and combination of them. The influence of surface roughness and $\mathrm{TiO}_{2}$ coating method for Ti rod on bioactivity was investigated by an in-vivo animal experiment [1]. In addition, a research work was performed to investigate the effect of surface roughness distribution and $\mathrm{TiO}_{2}$ coating on bioactivity of bone screw [2]. In these researches, it was found that the combination of surface roughness affected bioactivity on the surface of metallic implant. As the results, it is thought we can control the bioactivity with surface asperity which is created with AM. In the case of implant surface made with electron beam melting (EBM), the fabricated surface asperity was compared with that is manufactured with machining, and there was no significant difference. However, there was a difference of bioactivity in in-vivo experiment performed with rabbit femur [3]. The bioactivity on the surface fabricated by SLM should be investigated, because the surface roughness fabricated by SLM and EBM is different. The surface roughness depends on the hatching spacing and scanning speed in the case of AlSi10Mg with SLM [4]. 
In our previous report, we investigated the influence of surface asperity of implant model on bioactivity by means of an in-vivo experiment [5]. The square pole implant models were fabricated by SLM and they were introduced into the rat femurs. The model has 3 types of surfaces: top surface, side surface and polished surface. As the result, the bioactivity of the polished surface was the highest and as-fabricated surfaces, top and side surfaces, were not good in 2 weeks after the implantation. New bone was formed faster on the polished surface more than that on the other surfaces.

In this study, the bioactivity of the implant models was investigated in 8 weeks after implantation and compared with the result of 2-weeks-raised rats. Sliced samples of the rat femurs with an implant were stained and observed with optical microscope. The osteoconductivity was evaluated by bone-implant contact area ratio $R_{B-I}$ in the observed images of the samples. The influences of the surface roughness and implantation period on the $R_{B-I}$ was researched.

\section{Experimental methods}

\subsection{Processing conditions of implant model}

Square pole implant models were fabricated by SLM. They were made by using a hybrid metal 3D printer of LUMEX Avance-25 produced by Matsuura Machinery Corporation. This machine has a powder bed fusion system with a $\mathrm{Yb}$ fiber laser oscillator. The gas atomized Ti-6Al-4V powders were used and the particle size is $45 \mu \mathrm{m}$ or less. Its chemical composition is shown in Table 1.

The cross-sectional shape of the implant model was $\square 0.6 \mathrm{~mm}$ in design value and the actual cross-sectional dimension was $\square 0.83 \mathrm{~mm}$. The models were made for animal test with IGS-rat. The shape must be produced small because of the size of their femur.

Fig. 1 shows the shape of the implant model. The length of the implant models was $40 \mathrm{~mm}$. Seven pieces of implant model were fabricated in argon atmosphere on base plates of $10 \mathrm{~mm}$ in thickness and $\square 64 \mathrm{~mm}$. The fabrication height was $2.6 \mathrm{~mm}$ including the basement which height was $2 \mathrm{~mm}$. The models were separated from the base plate by means of a wire electric discharger machine. The bottom surface of the models were polished by emery papers to make the surface flat and relieve their deterioration layer.

In order to produce small products with SLM, processing conditions were change from the standard conditions. In this study, the laser spot diameter was $\Phi 0.1 \mathrm{~mm}$, this value was smaller than the usual value. The processing conditions are shown in Table 2. The scanning direction of the laser path was parallel to the longitudinal direction of the models.

An implant model has 3 types of surface asperity: top surface, side surface and bottom (polished) surface which are created during the fabrication (Fig. 2). In our previous report, we measured surface roughness of each surface. Table 3 shows the surface roughness. There was a large surface asperity on the top surface due to the bead shape. The side surface had small unevenness, because the powder was sintered. The polished surface was flat. We focused on the difference of the typical surface asperity of SLM.

Table 1. Chemical composition of Ti- $6 \mathrm{Al}-4 \mathrm{~V}$ powder (wt $\%)$.

\begin{tabular}{llllllll}
\hline $\mathrm{Al}$ & $\mathrm{V}$ & $\mathrm{Fe}$ & $\mathrm{C}$ & $\mathrm{N}$ & $\mathrm{H}$ & $\mathrm{O}$ & $\mathrm{Ti}$ \\
\hline $5.5-6.75$ & $3.5-4.5$ & 0.40 & 0.08 & 0.05 & 0.015 & 0.25 & Bal. \\
\hline
\end{tabular}

Table 2. Processing conditions of implant model. 


\begin{tabular}{ll}
\hline Power of laser [W] & 120 \\
\hline Layer thickness [mm] & 0.05 \\
\hline Spot diameter [mm] & 0.1 \\
\hline Hatch spacing [mm] & 0.05 \\
\hline Scanning speed [mm/s] & 800 \\
\hline
\end{tabular}

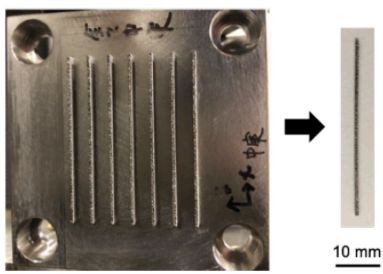

Fig. 1. Implant model of Ti-6Al-4V fabricated by SLM.
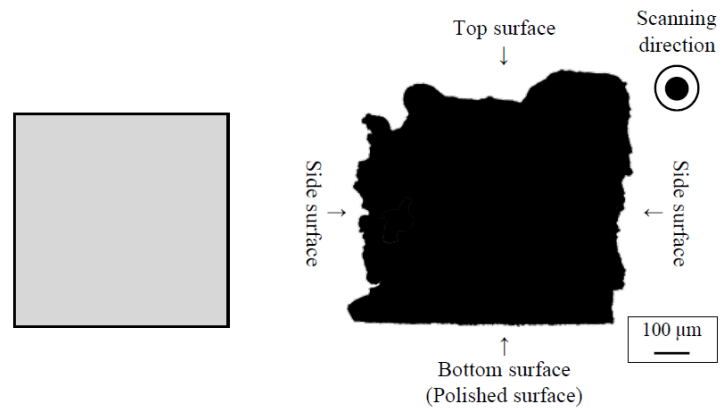

Fig. 2. Cross-sectional view of implant model of Ti-6Al-4V (Left; CAD model, Right; actual shape).

Table 3. Surface roughness of implant model in parallel to the longitudinal direction.

\begin{tabular}{lcc}
\hline & $\mathrm{Rz}[\mu \mathrm{m}]$ & $\mathrm{Rsm}[\mu \mathrm{m}]$ \\
\hline Top surface & 6.3 & 19.5 \\
\hline Side surface & 64.2 & 20.5 \\
\hline Polished surface & 2.0 & 15.5
\end{tabular}

\subsection{Implantation test}

The implant models were sterilized by autoclaving and then inserted into femur of 6-week-old IGS rat. Table 4 shows the implantation conditions. The surgical procedure of the implantation is shown in Fig. 3. First, the rats were anesthetized. Next, a hole with a diameter of $1.2 \mathrm{~mm}$ was drilled in the bone medullary cavity of the femur in the longitudinal direction with an injection needle from the hip joint side to the knee joint side, because the implant models were thin and weak. The model was inserted into the hole from the knee joint side at the same time as the injection 
Influence of Implantation Period on Bioactivity on the Surface of Ti-6Al-4V Implant Mod...

needle was pulled out. Finally, the end of the model protruding from the skin on the hip joint side was cut. After the operation, 3 rats were raised for 2 weeks and the other 3 rats were raised for 8 weeks.

Table 4. Implantation conditions of implant model.

\begin{tabular}{ll}
\hline Rat & Crl:CD IGS rat, 6-week-old, male \\
\hline Installation & Both feet, femur \\
\hline Period & 2,8 weeks \\
\hline Specimen & Intramedullary nail \\
\hline Sliced sample & Thickness: $40 \mu \mathrm{m}$, staining: toluidine blue \\
\hline
\end{tabular}

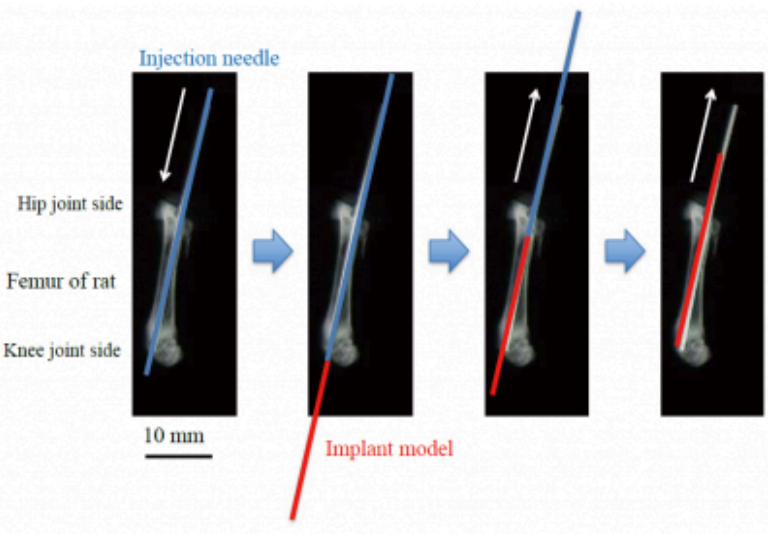

Fig. 3. Implantation procedure of Ti-6Al-4V implant model into femur of rat.

\subsection{Bioactivity evaluation}

Fig. 4 shows an example of cross-sectional view of a bone with implantation of a pure Ti rod. The bone consists of cortical bone and cancellous bone. The cortical bone is the dense outer surface of bone, which is composed of hard tissue and carries the mechanical load. We evaluated the osteoconductivity on each surface of the implant models.

In 2 or 8 weeks after the implantation, the implant model and surrounding bone tissue were sliced around the distal metaphysis of the femur in perpendicular to the longitudinal direction. The sliced samples were polished with emery paper to a thickness of $40 \mu \mathrm{m}$ and stained with toluidine blue. The contact length of hard tissue on a surface was evaluated on an image obtained with optical microscope.

The osteoconductivity was estimated by the bone-implant contact ratio $\left(R_{B-I}\right)$ defined by equation (1).

$$
R_{B-I}(\%)=\frac{\text { Hard tissue contact length }}{\text { Each surface length }} \times 100
$$




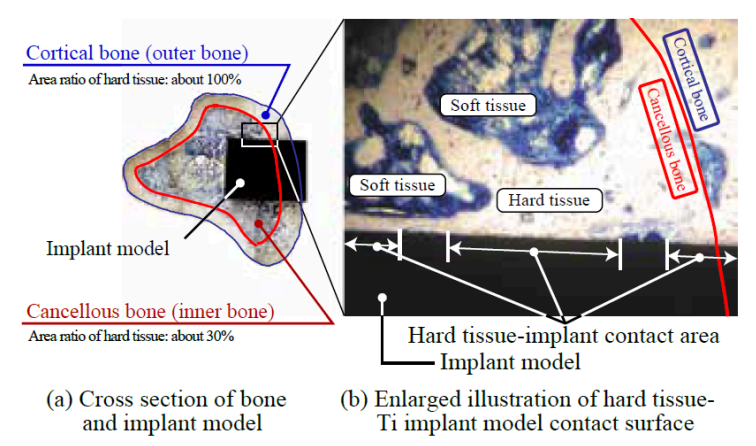

Fig. 4. Sliced sample of bone with implant model.

\section{Results and discussion}

\subsection{Observation of biological response with optical microscope}

Fig. 5 shows sliced samples of diaphyseal of femur with Ti-6Al-4V implant model in the case of 8-week-raised rats. The black area is the cross-section of the implant model and the white area around the implant model is hard tissue of cortical bone. The figure shows that the surfaces fabricated by SLM were acceptable in bone tissue as in the case of 2-week-raised rats. There were generally few signs of inflammation. A large part of the surface of the implant model was in direct contact with hard tissue of the cortical bone. The contact length is especially long in the case of the top surface. Although hard tissue was formed near the polished surface, there are some areas where the hard tissue directly contacted with the surface. In the case of the side surface, it was observed that the hard tissue was not in contact with the bottom of the valley. There were some voids inside of the implant models and the hard tissue formation was observed in the voids.

\subsection{Influence of implantation period on osteoconductivity}

Fig. 6 shows the relationship between bone-implant contact ratio $R_{B-I}$ and each surface of the implant model in the cortical bone. The outer surface of the cortical bone was neglected from the calculation of the $R_{B-I}$. In the case of 2-weeks-raised rats, the $R_{B-I}$ on the top surface was the lowest and that on the polished surface was the highest [5]. The polished surface has osteoconductivity in the cortical bone of the rat femur in short-term after the implantation. However, in the case of 8-weeks-raised rats, the $R_{B-I}$ on the top surface was the highest and that of the polished surface was the lowest. The $R_{B-I}$ on the polished surface decreased with increase of the implantation period. In the case of the top surface, there was a large difference in the $R_{B-I}$. The $R_{B-I}$ was high at about $90 \%$ in 8 weeks after implantation, even though the $R_{B-I}$ was low at about $10 \%$ in 2 weeks. The $R_{B-I}$ of the polished surface in the case of 8 -weeks-raised rats was higher than the case of top and side surface in 2-weeks-raised rats. If the implant model is required for new bone formation in a short period, the surface of the implant model should be polished. If the implant model is used for a long time, the $R_{B-I}$ on the side surface should be advanced. The $R_{B-I}$ on the side surface was about $50 \%$ in the case of 2 -weeks-raised rats. The $R_{B-I}$ was over $70 \%$ in 8 weeks after implantation and this was better than that on the polished surface in 2 and 8 weeks after implantation. 


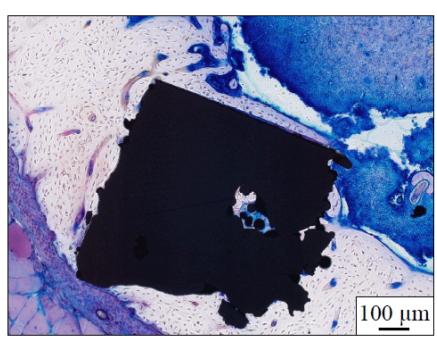

(a) 8 weeks-No. 1

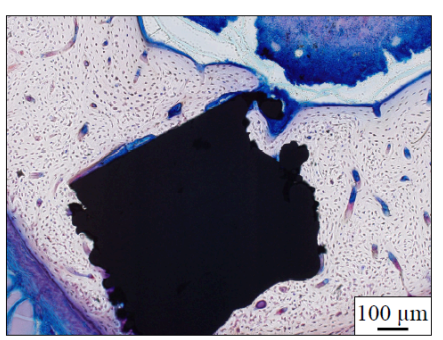

(b) 8 weeks-No. 2

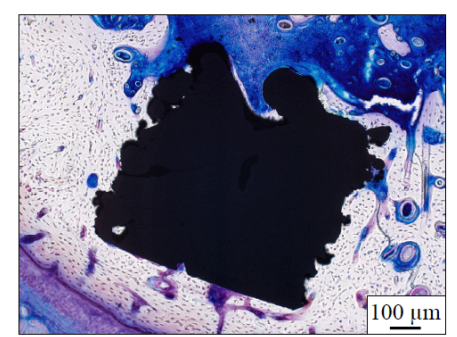

(c) 8 weeks-No. 3

Fig. 5. Sliced samples of rat femurs in 8 weeks after the implantation.

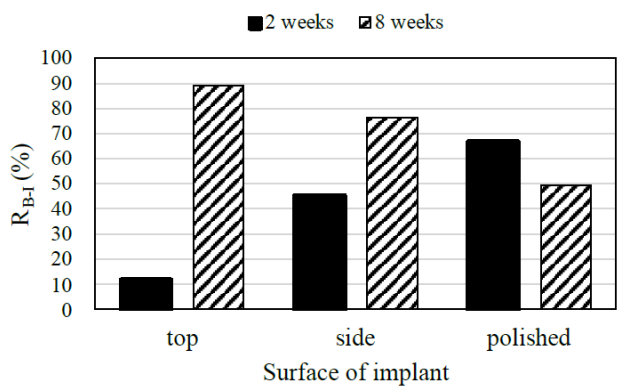

Fig. 6. The relation between $R_{B-I}$ and surface of Ti-6Al-4V implant model fabricated by SLM.

\section{Conclusions}

The Ti-6Al-4V implant models were manufactured with 3 types of surfaces: top surface, side surface and polished surface. They were fabricated by additive manufacturing method with selective laser melting. The implant models were introduced into rat femurs and the influence of the implantation period on bone-implant contact ratio $R_{B-I}$ was evaluated in 2 and 8 weeks after implantation. As the result, in the case of 2 -weeks-raised rats, the $R_{B-I}$ was the lowest on the top surface and that of the polished surface was the highest. However, when the implantation period exceeded 8 weeks, the trend has reversed, and the $R_{B-I}$ on the top surface would be the highest, and that on the polished surface was lowest.

\section{Bibliography}

[1] Yoshida, Y. Kuroda, K. Ichino, R. Hayashi, N. Ogihara, N. Nonaka, Y. Influence of surface properties on bioactivity and pull-out torque in cold thread rolled Ti rod-Development of bioactive metal-forming technology. CIRP Annals Manufacturing Technology, 2012, 61, 579-582

[2] Yoshida, Y. Kuroda, K. Ichino, R. Hayashi, N. Ogihara, N. Nonaka, Y. Development of bioactivity and pull-out torque control technology on Ti implant surface and its application for cold thread rolled bone screw. Procedia Engineering, $2014,81,340-345$

[3] Thomsen, P. Malmstrom, J. Emanuelsson, L. Rene, M. Snis, A. Electron Beam-Melted, Free-Form-Fabricated Titanium Alloy Implants: Material Surface Characterization and Early Bone Response in Rabbits. Journal of Biomedical Materials Research Part B: Applied Biomaterials, 2008, 90 B (1), 35-44 
[4] Han, X. Zhu, H. Nie, X. Wang, G. Zeng, X. Investigation on Selective Laser Melting AlSi10Mg Cellular Lattice Strus: Molten Pool Morphology, Surface Roughness and Dimensional Accuracy. Materials, 2008, 11, 392

[5] Kutsukake, A. Yoshida, Y. Influence of Surface Asperity Made by Selective laser Melting Additive Manufacturing on Bioactivity in Rat Femur. Procedia Manufacturing, 2020, 47, 1101-1105

PDF automatically generated on 2021-05-20 06:37:26

Article url: https://popups.uliege.be/esaform21/index.php?id=4350

published by ULiège Library in Open Access under the terms and conditions of the CC-BY License (https://creativecommons.org/licenses/by/4.0) 